\title{
Mid-infrared images of the massive star forming region $\mathrm{W} 75 \mathrm{~N}^{\star, \star \star}$
}

\author{
P. Persi ${ }^{1}$, M. Tapia ${ }^{2}$, and H. A. Smith ${ }^{3}$ \\ ${ }^{1}$ Istituto Astrofisica Spaziale e Fisica Cosmica, INAF, via fosso del cavaliere 100, 00133 Roma, Italy \\ e-mail: persi@rm.iasf.cnr.it \\ 2 Instituto de Astronomía, UNAM, Apartado Postal 877, 22830 Ensenada, BC Mexico \\ 3 Harvard Smithsonian Center for Astrophysics, 60 Garden Street, Cambridge, MA 02138, USA
}

Received 15 April 2005 / Accepted 22 August 2005

\section{ABSTRACT}

An infrared study that includes ground-based mid-infrared images between 8.7 and $18.7 \mu \mathrm{m}$ and IRAC images at 3.6, 4.5, 5.8 and 8.0 $\mu \mathrm{m}$ of the W75 N massive star forming region is presented. The $12.5 \mu \mathrm{m}$ image shows the presence of four mid-infrared sources in the region W75 N(B), three of which have bright near-infrared counterparts, IRS 1, IRS 2 and IRS 3, all with significant excess emission at $\lambda>2.0 \mu \mathrm{m}$. IRS 2 has a steep energy distribution and the computed infrared luminosity is consistent with the presence of a young B3 star. The observed IRAC colors of IRS 3 indicate that this source is a Class II intermediate mass young star, consistent with its infrared energy distribution and luminosity. The fourth, newly discovered, mid-infrared source appears coincident with the ultracompact HII region VLA 3, and is located within the millimeter core MM 1. We derived a luminosity of $\sim 750 L_{\odot}$ and a visual extinction $A_{\mathrm{V}} \simeq 90$ for this source. From the IRAC images, we detected 75 sources in an area of $120^{\prime \prime} \times 120^{\prime \prime}$ centered in W75 N. At least 25 of these sources are associated with the molecular cloud and form a young stellar cluster as shown in the IRAC two-color and the $H-K_{\mathrm{s}}$ versus $K_{\mathrm{s}}-$ [3.6] diagrams.

Key words. stars: formation - infrared: stars - ISM: clouds - ISM: HII regions

\section{Introduction}

The massive star-forming region $\mathrm{W} 75 \mathrm{~N}$ is located at a distance of $2 \mathrm{kpc}$ and is, together with DR 21, part of the Cygnus $\mathrm{X}$ complex of dense molecular clouds. Haschick et al. (1981) detected at $6 \mathrm{~cm}$ an ultracompact HII (UC HII) region named W75 N(B), and two more extended HII regions, W75 N(A) and $\mathrm{W} 75 \mathrm{~N}(\mathrm{C})$. Observations at $3.6 \mathrm{~cm}$ with $0.5^{\prime \prime}$ resolution by Hunter et al. (1994) show that W75 N(B) is resolved into three very small diameter $\left(\leq 0.1^{\prime \prime}\right)$ radio continuum sources: $\mathrm{Ba}, \mathrm{Bb}$ and $\mathrm{Bc}$. Two of these, $\mathrm{Ba}$ and $\mathrm{Bb}$, were also detected at $1.3 \mathrm{~cm}$ (with a $0.1^{\prime \prime}$ resolution) by Torrelles et al. (1997) (who named these VLA 1 and VLA 3) along with another fainter and more compact HII region, VLA 2. These UC HII regions are in a protostellar phase, based on the presence of $\mathrm{H}_{2} \mathrm{O}$ and/or OH masers (Baart et al. 1986, Hunter et al. 1994, Torrelles et al. 1997) in its close vicinity. At radio wavelengths, VLA 1 has a structure elongated approximately in the direction of the observed high-velocity three pc-scale bipolar outflow located close to the center of the nearby W75 N(B)

\footnotetext{
* Based on observations collected at the Observatorio Astronómico Nacional, San Pedro Mártir, B.C, Mexico, and with IRAC on board of Spitzer Space Telescope.

$\star \star$ The complete list of the source is only available in electronic form at http://www.edpsciences.org
}

(Hunter et al. 1994; Shepherd et al. 2003) while VLA 2 shows a symmetric roundish shape. A detailed VLBI study of the water maser cluster morphology and kinematics in the vicinity of these two UC HII regions by Torrelles et al. (2003) showed remarkably different outflow ejection geometries among them. This is a surprising result given that both objects are separated by only $1400 \mathrm{AU}$ and share the same environment. The physical parameters of the ionized gas in VLA 1, VLA 2, VLA 3, and $\mathrm{Bc}$, as well as the more evolved W75 N(A) are all consistent with each being powered by a ZAMS star of spectral type between B0 and B2 (Shepherd et al. 2004).

Four compact millimeter cores were observed by Shepherd (2001) within a radius of 10" (MM 1-4). Three UC HII regions are located within the MM 1 core. Another extended millimeter core (MM 5) is located $\sim 30^{\prime \prime}$ to north of MM 1 and is coincident with the extended HII region W75 N(A). Multiple outflows have been identified originating from the cluster of UC HII regions and millimeter cores (Davis et al. 1998; Shepherd et al. 2003).

Two reflection nebulosities are seen in the near-infrared: one associated with $\mathrm{W} 75 \mathrm{~N}(\mathrm{~A})$ and the other at the position of W75 N(B) (Moore et al. 1991b; Shepherd et al. 2003). In addition, three bright sources (IRS1, IRS2 and IRS3) were detected with significant excess emission at $2.2 \mu \mathrm{m}$, consis- 

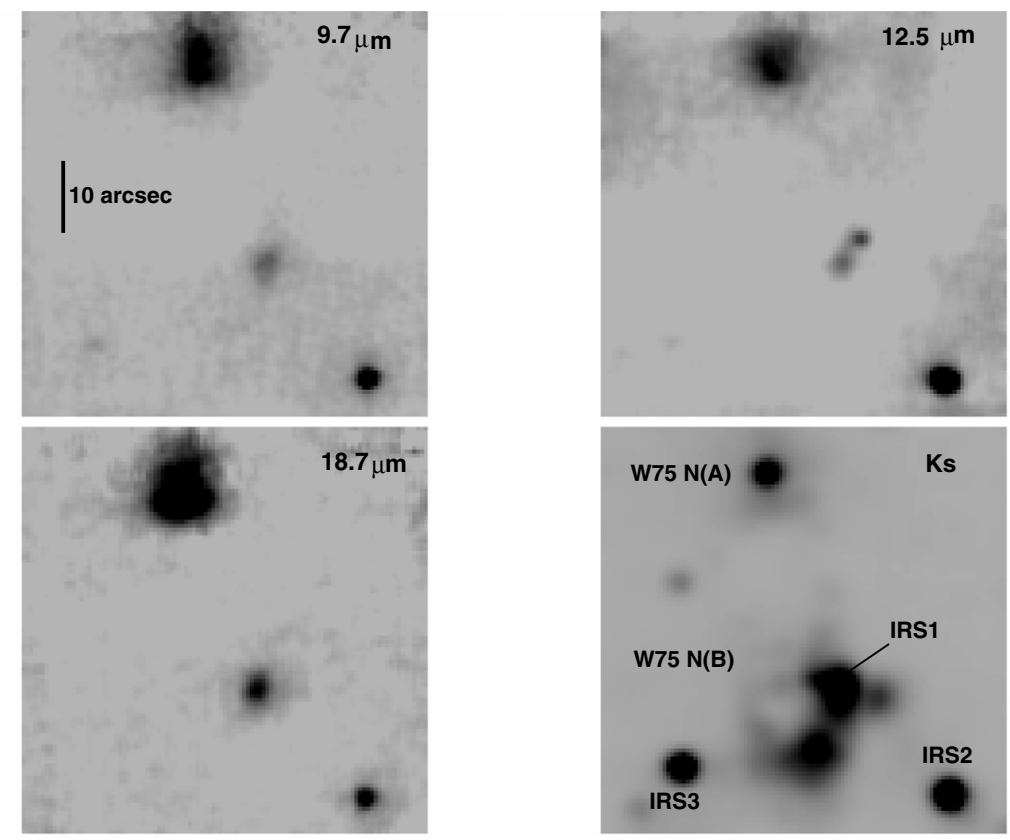

Fig. 1. $58^{\prime \prime} \times 58^{\prime \prime}$ images at 9.7, 12.5 and $18.7 \mu \mathrm{m}$ of $\mathrm{W} 75 \mathrm{~N}$ centered around $\mathrm{W} 75 \mathrm{~N}(\mathrm{~B})$. For comparison, the $2 \mathrm{MASS} K_{\mathrm{s}}$ image of the region is also shown with the same scale. The center is at $\alpha=20^{\mathrm{h}} 38^{\mathrm{m}} 37^{\mathrm{s}} .2 ; \delta=+42^{\circ} 37^{\prime} 37^{\prime \prime}$ (J2000). North is to the top and east to the left.

tent with the presence of circumstellar dust. IRS1, IRS2 as well as W75 N(A) were also detected at 10 and $20 \mu \mathrm{m}$ (Moore et al. 1991a). In particular, the near-infrared hydrogen recombination lines $\operatorname{Br} \gamma, \operatorname{Br} \alpha$ and $\operatorname{Pf} \gamma$ observed in in the direction of IRS2 indicate that this young massive star is undergoing significant mass loss (Moore et al. 1991b). Except for MM 5, none other of the millimeter sources had a known infrared counterpart, until now, with the discovery of the mid-infrared source associated with MM 1 described in Sect. 3.4.

All these observations show that $\mathrm{W} 75 \mathrm{~N}$ is a very complex star forming region with a cluster of intermediate and highly massive stars with a spread in ages between the oldest and youngest stars of $0.1-5 \times 10^{6} \mathrm{yr}$ (Shepherd et al. 2004).

To investigate the nature of the young objects in $\mathrm{W} 75 \mathrm{~N}$, we obtained mid-infrared images between 8.7 and $18.7 \mu \mathrm{m}$. We compared the mid-infrared ground-based observations with the images of the region taken with the Infrared Array Camera (IRAC; Fazio et al. 2004) aboard the Spitzer Space Telescope (Werner et al. 2004). W75 N was detected by as part of large scale observations of the complex massive star forming region DR 21 (Marston et al. 2004).

\section{Observations}

\subsection{Ground-based mid-infrared images}

Mid-infrared images between 8.7 and $18.7 \mu \mathrm{m}$ of $\mathrm{W} 75 \mathrm{~N}$ were taken during three observing runs on September 2000, November 2001 and September 2003 with the mid-infrared camera CID (Salas et al. 2003) on the $2.1 \mathrm{~m}$ telescope of the Observatorio Astronómico Nacional at San Pedro Mártir, Baja California, Mexico. This camera is equipped with a Rockwell $128 \times 128$ pixel Si:As BIB detector array and delivers an effec- tive scale of $0.55^{\prime \prime} /$ pix. The images were taken in the standard chop-nodding technique to remove the sky and telescope emission background.

The narrow-band images at 8.7, 9.7, 11.7 and $12.5 \mu \mathrm{m}$ from September 2000 are centered at the position of IRS2 and cover an area of 58" $\times 58^{\prime \prime}$. During the November 2001 and September 2003 runs, the images at 9.7, 12.5 and $18.7 \mu \mathrm{m}$ were centered on the W75 N(B) in order to also include W75 N(A). The images at $18.7 \mu \mathrm{m}$ were gathered during the September 2003 run. Figure 1 shows these images and the $K_{\mathrm{s}}$ image from the Two Micron All Sky Survey (2MASS) for comparison.

The standard stars $\alpha$ Lyr, $\beta$ Peg, $\gamma$ Aql and $\mu$ Cep were observed at similar air-masses for flux calibration. These were also used to measure the point-spread function at each wavelength. The mean PSF ranged from $\sim 1.6-1.8^{\prime \prime}$ (FWHM) at shorter wavelengths, and $\sim 2.6^{\prime \prime}$ at $18.7 \mu \mathrm{m}$. These are slightly larger than the corresponding Airy disk sizes. The on-source integration times were $570 \mathrm{~s}$ at $8.7 \mu \mathrm{m}, 720 \mathrm{~s}$ at 9.7 and $12.5 \mu \mathrm{m}$ and $860 \mathrm{~s}$ at $18.7 \mu \mathrm{m}$. The derived detection limits, which depend on the sky quality of the night, are $\sim 0.4 \mathrm{Jy}, 0.6 \mathrm{Jy} 0.5 \mathrm{Jy}$ and $5.0 \mathrm{Jy}(1 \sigma)$, respectively.

Five mid-infrared sources were detected at $12.5 \mu \mathrm{m}$. Four of these (IRS 1, IRS 2, IRS 3, and MM 1) are within the area of W75 N(B) (see Fig. 1), while the extended mid-infrared source to the NE corresponds to the HII region W75 N(A). Astrometry of our images were obtained from the 2MASS coordinates of the identified sources in the field.

The photometry of the mid-infrared sources was performed with the DAOPHOT package within IRAF (Stetson 1987). We used an aperture of $3.3^{\prime \prime}$ for the point-like sources, and $6^{\prime \prime}$ for the diffuse source. The coordinates, the flux densities, the $1 \sigma$ statistical errors, and the identifications of the mid-infrared sources, are given in Table 1. 
Table 1. Coordinates and mid-infrared photometry of the sources in $\mathrm{W} 75 \mathrm{~N}$.

\begin{tabular}{|c|c|c|c|c|c|c|c|c|c|}
\hline \multirow[t]{2}{*}{ Source } & \multirow[t]{2}{*}{ Date } & $\alpha(J 2000)$ & $\overline{\delta(J 2000)}$ & \multirow{2}{*}{$\begin{array}{l}{[8.7]} \\
(\mathrm{Jy})\end{array}$} & \multirow{2}{*}{$\begin{array}{l}\begin{array}{l}9.7] \\
(\mathrm{Jy})\end{array} \\
\end{array}$} & \multirow{2}{*}{$\begin{array}{l}{[11.7]} \\
(\mathrm{Jy})\end{array}$} & \multirow{2}{*}{$\begin{array}{l}{[12.5]} \\
\text { (Jy) }\end{array}$} & \multirow{2}{*}{$\begin{array}{l}{[18.7]} \\
\text { (Jy) }\end{array}$} & \multirow[t]{2}{*}{ ID. } \\
\hline & & $\mathrm{h} \quad \mathrm{m} \quad \mathrm{s}$ & $\circ \quad$ & & & & & & \\
\hline IRS 2 & $9 / 17 / 00$ & 203835.4 & 423713.7 & $7.5(0.5)$ & $4.0(0.6)$ & $9.9(0.7)$ & $11.4(1.0)$ & & \\
\hline " & $11 / 7 / 01$ & & & & $5.5(0.7)$ & & $9.8(0.5)$ & & \\
\hline$"$ & $9 / 30 / 03$ & & & & & & $14.1(2.8)$ & $21.2(4.1)$ & \\
\hline MM 1 & $9 / 17 / 00$ & 203836.5 & 423733.6 & & & $1.1(0.3)$ & $2.1(0.4)$ & & VLA $3(\mathrm{Bb})$ \\
\hline$"$ & $11 / 7 / 01$ & & & & & & $2.0(0.2)$ & & \\
\hline$"$ & $9 / 30 / 03$ & & & & & & $2.6(0.5)$ & & \\
\hline IRS 1 & $9 / 17 / 00$ & 203836.6 & 423730.7 & & & $1.9(0.3)$ & $2.8(0.4)$ & & \\
\hline$"$ & $11 / 7 / 01$ & & & & $2.4(0.5)$ & & $2.3(0.3)$ & & \\
\hline$"$ & $9 / 30 / 03$ & & & & & & $3.5(0.7)$ & $26.5(5.1)$ & \\
\hline IRS 3 & $11 / 7 / 01$ & 203838.9 & 423718.4 & & $0.8(0.3)$ & & $0.6(0.2)$ & & \\
\hline W75N(A) & $11 / 7 / 01$ & 203837.6 & 423758.6 & & $16.6(1.4)$ & & $12.9(0.7)$ & & \\
\hline$"$ & $9 / 30 / 03$ & & & & & & $18.8(3.8)$ & $153.4(23.6)$ & \\
\hline
\end{tabular}

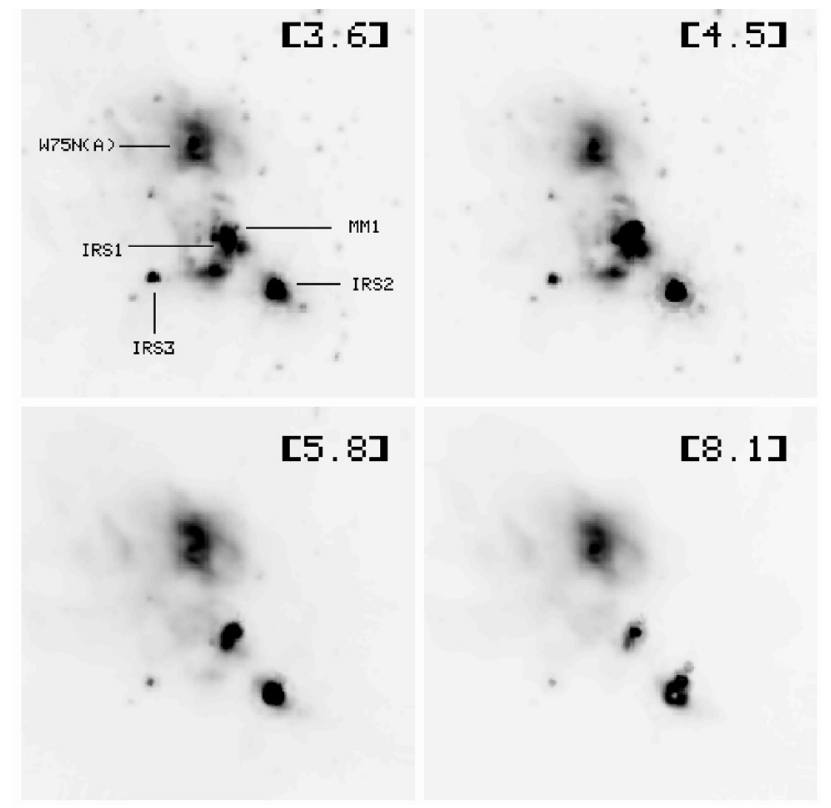

Fig. 2. $120^{\prime \prime} \times 120^{\prime \prime}$ IRAC images at 3.6, 4.5, 5.8, and $8.0 \mu \mathrm{m}$ of W $75 \mathrm{~N}$ centered as in Fig. 1. North is the top and east to the left. The blobs to the NW of the saturated IRS 2 are electronic ghosts in the $8 \mu \mathrm{m}$ image.

\subsection{Spitzer images}

The images at 3.6, 4.5, 5.8 and $8.0 \mu \mathrm{m}$ of $\mathrm{W} 75 \mathrm{~N}$ presented here are sections of the original IRAC images of the star forming region DR21 observed during the science verification period of the Spitzer satellite. Preliminary results of the whole DR21 region were reported by Marston et al. (2004). Figure 2 shows the IRAC images at the four wavelength bands corresponding to an area of $120^{\prime \prime} \times 120^{\prime \prime}$ centered closed to W75 N(B). The IRAC images are dominated by diffuse emission at all wavelengths. The positions of the mid-infrared sources detected from the ground are indicated on the $3.6 \mu \mathrm{m}$ image in Fig. 2. Source IRS 2 is partially saturated at all wavelengths, while IRS 1 is slightly extended and is also partially saturated at 4.5, 5.8 and $8.0 \mu \mathrm{m}$. The newly detected midinfrared source associated with MM 1 appears point-like on our 11.7 and $12.5 \mu \mathrm{m}$ images, and is seen well separated from
Table 2. Observed IRAC flux densities of MM 1 and IRS 3.

\begin{tabular}{lllll}
\hline \hline Source & $\begin{array}{l}{[3.6]} \\
(\mathrm{Jy})\end{array}$ & $\begin{array}{l}{[4.5]} \\
(\mathrm{Jy})\end{array}$ & $\begin{array}{l}{[5.8]} \\
(\mathrm{Jy})\end{array}$ & $\begin{array}{l}{[8.0]} \\
(\mathrm{Jy})\end{array}$ \\
\hline MM 1 & 0.018 & 0.19 & 0.23 & 0.67 \\
IRS 3 & 0.14 & 0.16 & 0.20 & 0.35 \\
\hline
\end{tabular}

IRS 1. Finally, IRS 3 was unresolved by IRAC. Table 2 reports the observed IRAC flux densities of these two unsaturated sources.

Source finding and photometry from the Spitzer satellite (pipeline version S10.0.1) released $2 \times 2$-magnified images of the region were performed using DAOPHOT package within IRAF. An aperture radius of 3 pixels $\left(\sim 2^{\prime \prime}\right)$ and a sky annulus extending from 3 to 7 pixels were used. We applied the corresponding aperture corrections and the zero magnitude fluxes from the Spitzer/IRAC User Manual, v. 5.0, (277.5 Jy, $179.5 \mathrm{Jy}$, $116.6 \mathrm{Jy}$ and $63.1 \mathrm{Jy}$ for channels 1, 2, 3 and 4, respectively) in order to convert fluxes into magnitudes. Seventy-five sources were found at 3.6 and $4.5 \mu \mathrm{m}$ of which approximately $63 \%$ were also detected at 5.8 , and $35 \%$ also at $8.0 \mu \mathrm{m}$. Using the 2MASS All-Sky catalog of point sources, we identified 49 objects detected in both surveys. The spatial coincidence of the IRAC and 2MASS sources was always within $1^{\prime \prime}$. The complete list of these sources, including their positions and the IRAC and 2MASS photometry (from the Point Source Catalog) is reported in electronic form. We estimate a photometric accuracy of $\sim 0.25 \mathrm{mag}$ in all filters for those sources surrounded by bright nebulosities, especially in the 5.8 and $8.0 \mu \mathrm{m}$ bands, as discussed by Megeath et al. (2004). We were able to obtain more accurate, corrected flux values for the bright, partially sources by fitting the IRAC PSF to the wings of the profiles. Figure 3 shows and RGB-coded image composed of the IRAC 1,2 and 4 bands (3.6, 4.5 and $8 \mu \mathrm{m}$, respectively). The quite different IRAC colors of each of the main sources is evident, as will be discussed in the following sections. Figures 4 shows a blow-up of the extended source W75 N(A) in 3.6, 5.8, $8 \mu \mathrm{m}$ compared with the CID $18.7 \mu \mathrm{m}$ contours while Fig. 5 shows the details of the IRS 1/MM 1 region in the same three IRAC bands together with the CID $12.5 \mu \mathrm{m}$ contours. The positions and sizes of the millimeter sources and a $2.12 \mu \mathrm{m}$ 


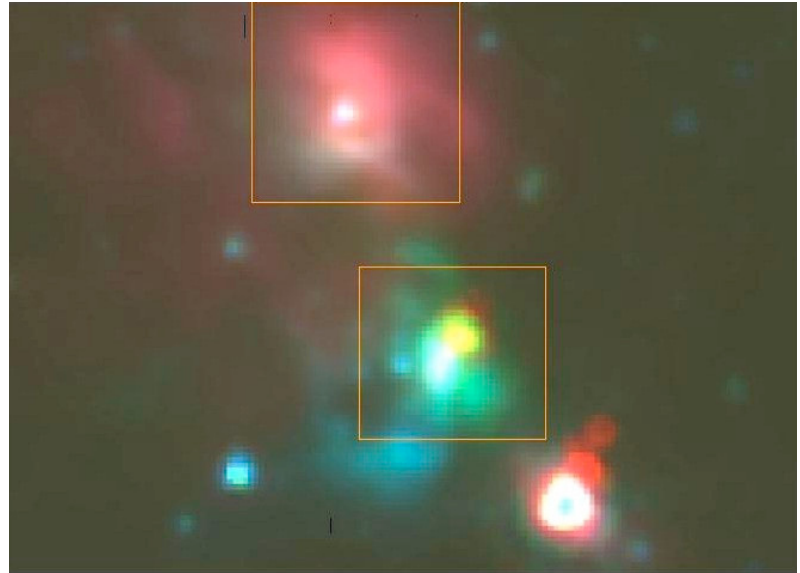

Fig. 3. $90^{\prime \prime} \times 66^{\prime \prime}$ RGB- coded image of W75 $\mathrm{N}$ taken with IRAC. $3.6 \mu \mathrm{m}$ is blue, $4.5 \mu \mathrm{m}$ is green and $8.0 \mu \mathrm{m}$ is red. The center is the same as in Fig. 1. North is the top and east to the left. The red blobs to the NW of the saturated IRS 2 are electronic ghosts in the $8 \mu \mathrm{m}$ image. The rectangles mark the expanded sections displayed in Figs. 4 and 5.

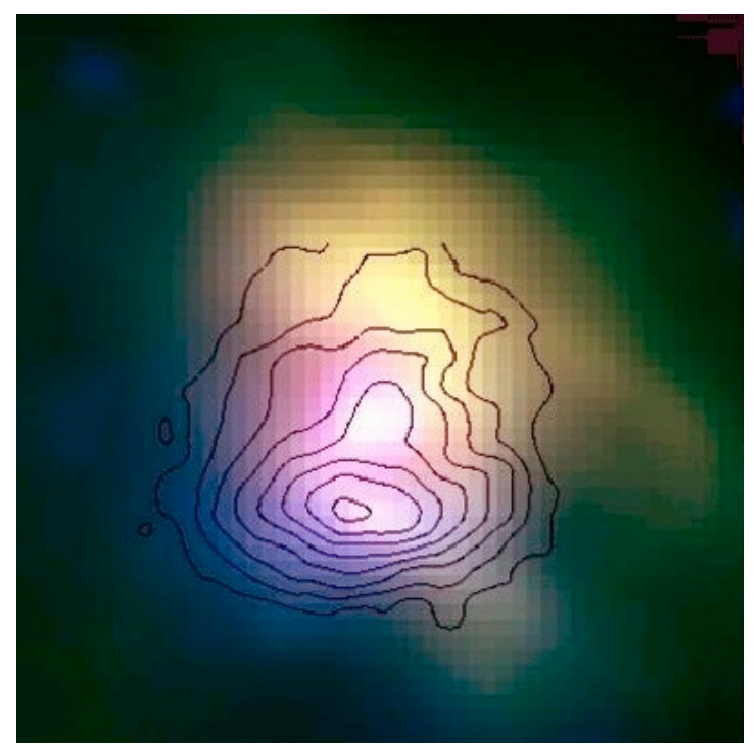

Fig. 4. RGB-coded image of W75 N(A) taken with IRAC $4.5 \mu \mathrm{m}$ is blue, $5.8 \mu \mathrm{m}$ is green and $8.0 \mu \mathrm{m}$ is red. The contours are from the $18.7 \mu \mathrm{m}$ CID image. The field size is $28^{\prime \prime} \times 26^{\prime \prime}$ and the center is at $\alpha=20^{\mathrm{h}} 38^{\mathrm{m}} 36.6 ; \delta=+42^{\circ} 38^{\prime} 31^{\prime \prime}$ (J2000). North is the top and east to the left.

$\mathrm{H}_{2}$ emission knot (from Shepherd 2003) are schematically indicated.

\section{Discussion}

\subsection{IRAC and near-infrared color-color plots}

In order to study the nature of the sources detected by IRAC, we analyzed the [3.6]-[4.5] versus [5.8]-[8.0] color diagram reported in Fig. 6 (left panel). According to Allen et al. (2004) and Megeath et al. (2004), sources within the box in Fig. 6 (left panel) are class II objects, while sources with ([3.6]-[4.5]) $\geq$ 0.8 and $([5.8]-[8.0]) \geq 1.1$ are classified Class I. Finally, a

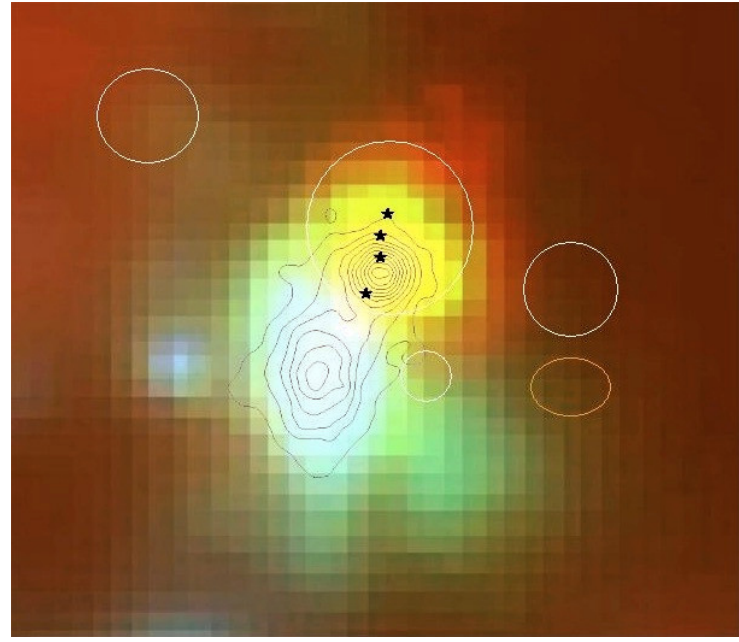

Fig. 5. RGB-coded image of W75 IRS 1/MM 1 taken with IRAC $4.5 \mu \mathrm{m}$ is blue, $5.8 \mu \mathrm{m}$ is green and $8.0 \mu \mathrm{m}$ is red. The contours are from the $12.5 \mu \mathrm{m}$ CID image. The black stars represent the UC HII regions (fron $\mathrm{N}$ to $\mathrm{S}$ ) VLA 1, VLA 2, VLA 3 and Bc. The large circles represent the $2.3 \mathrm{~mm}$ sources and the ellipse a knot of $\mathrm{H}_{2}$ emission (Shepherd et al. 2003). The field size is $22^{\prime \prime} \times 20^{\prime \prime}$ and the center is at $\alpha=20^{\mathrm{h}} 38^{\mathrm{m}} 37.8 ; \delta=+42^{\circ} 38^{\prime} 31^{\prime \prime}(\mathrm{J} 2000)$. North is the top and east to the left.

number of sources have $([5.8]-[8.0]) \geq 1.1$, but $([3.6]-[4.5]) \leq$ 0.8 . These IRAC colors are reminiscent of those of compact HII regions where the $7.7 \mu \mathrm{m}$ and $8.6 \mu \mathrm{m}$ "aromatic" emission features dominate the IRAC band 4. Many of these appear as knots of filaments on the $8.0 \mu \mathrm{m}$ image as indicated for each source in the last column (Notes) of Table 3. Jones et al. (2005) also reported these effects on the IRAC color-color diagram. The positions of the young stellar objects IRS 3 and MM 1 are also shown in the figure. The location MM 1 in the IRAC twocolor diagram identify this source as an extreme class I object, while IRS 3 is in the area of class II sources.

We analyzed also the $H-K_{\mathrm{s}}$ versus $K_{\mathrm{s}}-$ [3.6] diagram for the 32 sources detected in $H, K_{\mathrm{s}}$ and $3.6 \mu \mathrm{m}$ (Fig. 6, right panel). A significant fraction of the sources lie to the right of the reddening vector ${ }^{1}$ at distances $\Delta\left(K_{\mathrm{s}}-\right.$ [3.6]) at least three times the maximum individual photometric uncertainty (as measured while performing the PSF photometry with a minimum value of 0.1 , which is the estimated calibration error). These very young sources, listed in Table 3, exhibit significant near-infrared excesses, in most cases due to the presence of disks.

From the two color-color plots, the presence of a young stellar cluster composed by at least of 25 young stellar objects in W75 N is clearly inferred. This cluster includes several

\footnotetext{
1 We adopted a value of the reddening ratio $E_{H-K} / E_{K-L}=1.3 \mathrm{de}-$ termined by Tapia (1981) for a set of highly reddened field stars since the nominal effective wavelength of the original (Johnson's) $L$-band filter $\left(\lambda_{0}=3.5 \mu \mathrm{m}\right)$ is only slightly shorter than IRAC's channel 1 $\left(\lambda_{\circ}=3.55 \mu \mathrm{m}\right)$, a shift which is compensated by the effect of the telluric bands at $\lambda_{0}<3.4 \mu \mathrm{m}$. This is confirmed by the fact the value for the color index ratio that we used is almost identical to that derived $\left(E_{H-K} / E_{K-L}=1.26\right)$ by Indebetouw et al. (2005) from GLIPSE observations of reddened field stars.
} 

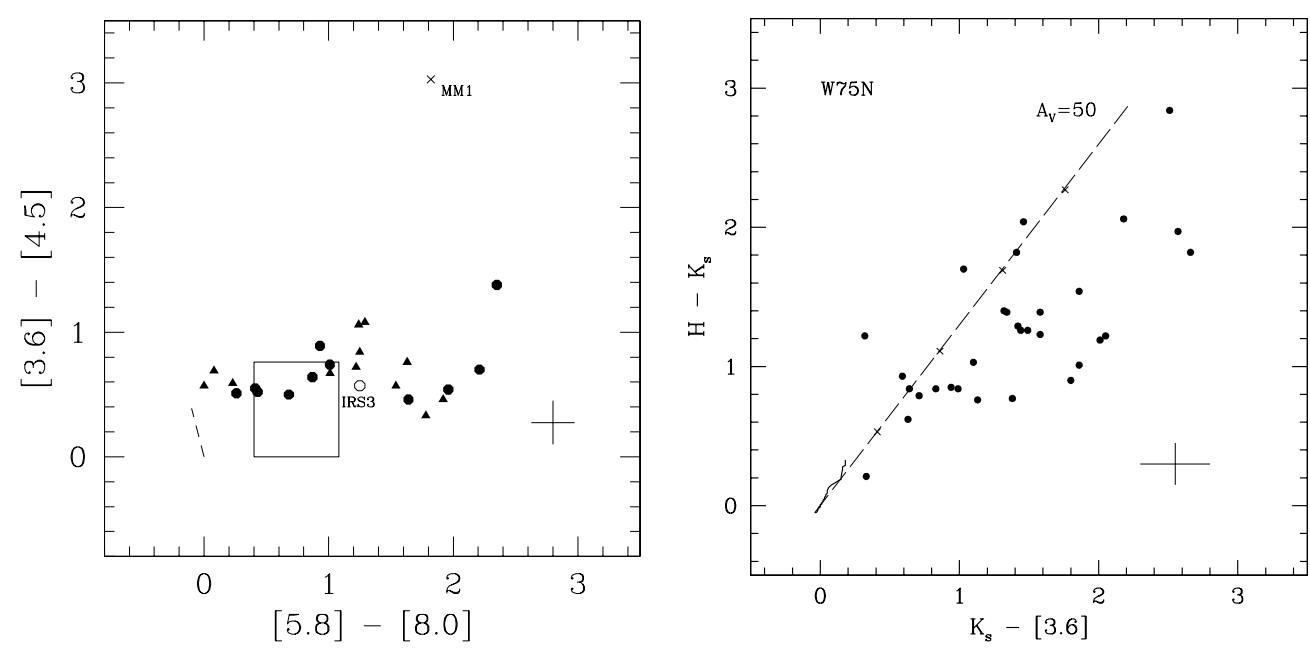

Fig. 6. Left panel IRAC color-color diagram of the sources observed in an area of $120^{\prime \prime} \times 120^{\prime \prime}$ in W75 N(B). The box delineates the approximate domain of class II sources (Megeath et al. 2004, Allen et al. 2004). Filled triangles represent sources not detected in the near-infrared, filled circles, those with 2MASS counterpart. MM 1 and IRS 3 have special symbols. Right panel $H-K_{\mathrm{s}}$ versus $K_{\mathrm{s}}-$ [3.6] plot for the 32 sources in common between IRAC and 2MASS. The continuous line marks the locus of the main sequence stars from Koornneef (1983) and the dashed line indicates the reddening vector with a slope of 1.3 as determined from highly reddened field stars by Tapia (1981). Tic marks are separated by $A_{\mathrm{V}}=10$. In both panels, the crosses show the maximum photometric errors.

Table 3. Coordinates and photometry from IRAC and 2MASS of sources with infrared excess.

\begin{tabular}{|c|c|c|c|c|c|c|c|c|c|c|}
\hline \multirow[t]{2}{*}{ ID } & $\alpha(J 2000)$ & $\delta(J 2000)$ & \multirow{2}{*}{$\begin{array}{l}{[3.6]} \\
\text { mag }\end{array}$} & \multirow{2}{*}{$\begin{array}{l}4.5] \\
\mathrm{mag}\end{array}$} & \multirow{2}{*}{$\begin{array}{l}5.8] \\
\mathrm{mag}\end{array}$} & \multirow{2}{*}{$\begin{array}{l}8.0] \\
\text { mag }\end{array}$} & \multirow{2}{*}{$\begin{array}{l}J \\
\text { mag }\end{array}$} & \multirow{2}{*}{$\begin{array}{l}H \\
\mathrm{mag}\end{array}$} & \multirow{2}{*}{$\begin{array}{l}K \\
\text { mag }\end{array}$} & \multirow[t]{2}{*}{ Notes } \\
\hline & $\mathrm{m} \quad \mathrm{s}$ & $\circ$ & & & & & & & & \\
\hline 1 & 203831.65 & 423833.5 & 12.18 & 11.46 & 10.68 & 9.46 & & & 15.14 & ps, clII \\
\hline 3 & 203832.36 & 423741.6 & 13.25 & 12.41 & 11.76 & 10.51 & & & & ps, clII \\
\hline 6 & 203833.15 & 42386.3 & 12.81 & 12.24 & 11.50 & 9.96 & & & 14.27 & psneb, HII \\
\hline 12 & 203833.62 & 423726.5 & 11.66 & 10.77 & 9.97 & 9.04 & & 16.14 & 14.32 & ps, clII \\
\hline 13 & 203833.64 & 423710.7 & 12.57 & 12.30 & & & & 15.77 & 14.58 & psneb, clII \\
\hline 14 & 203833.68 & 423652.1 & 11.39 & 10.87 & 10.21 & 9.78 & & 14.36 & 12.97 & ps, clII? \\
\hline 18 & 203834.23 & 423736.8 & 12.95 & 12.56 & & & & 15.10 & 14.33 & psneb, clII \\
\hline 23 & 203834.54 & 42386.7 & 12.47 & 11.71 & 10.92 & 9.29 & & & 14.94 & ps, HII \\
\hline 24 & 203834.64 & 42377.6 & 10.01 & 9.55 & 7.78 & 6.14 & & 15.43 & 14.14 & ps, HII \\
\hline 37 & 203835.81 & 423749.5 & 10.84 & 9.76 & 8.11 & 6.82 & & & & ps, clI \\
\hline 42 & 203836.42 & 423726.1 & 8.18 & 6.80 & 5.87 & 3.52 & & 13.53 & 10.69 & neb, clI? \\
\hline 44 & 203836.49 & 423831.3 & 12.94 & 12.27 & 11.10 & 10.09 & & & 14.63 & ps, clII \\
\hline 45 & 203836.54 & 423758.4 & 10.25 & 9.79 & 7.09 & 5.17 & & & 14.37 & neb, HII \\
\hline 47 & 203836.70 & 423728.8 & 7.60 & 6.22 & & & 14.11 & 11.84 & 9.78 & IRS 1 , sat b3,4 \\
\hline 49 & 203837.02 & 423735.2 & 9.40 & 8.29 & 8.02 & & & & & MM 3 \\
\hline 53 & 203837.76 & 423758.1 & 8.73 & 7.79 & 6.50 & 5.63 & 13.95 & 11.46 & 10.07 & W75N(A), clII \\
\hline 55 & 203837.82 & 423828.8 & 12.63 & 12.08 & 11.14 & 10.73 & & 15.90 & 14.68 & ps, clII? \\
\hline 57 & 203838.40 & 42371.1 & 13.17 & 13.06 & & & 16.70 & 15.06 & 14.30 & ps \\
\hline 59 & 203838.74 & 423836.2 & 13.26 & 13.54 & & & & 15.96 & 15.06 & ps \\
\hline 61 & 203838.88 & 423716.9 & 8.23 & 7.66 & 6.90 & 5.65 & 12.61 & 10.98 & 9.72 & IRS3, clII \\
\hline 62 & 203838.91 & 423742.6 & 10.27 & 9.53 & 8.93 & 7.92 & 17.08 & 13.67 & 12.13 & psneb,clII \\
\hline 67 & 203840.09 & 42388.0 & 11.36 & 11.14 & 10.51 & & 16.13 & 14.23 & 13.22 & psneb \\
\hline 73 & 203840.90 & 423838.9 & 12.66 & 12.33 & 10.27 & 8.49 & & & 14.60 & psneb, HII \\
\hline 74 & 203841.25 & 423738.9 & 12.39 & 11.33 & 9.23 & 7.99 & & & & psneb, clII \\
\hline
\end{tabular}

NOTES. clII: class II; clI: class I; ps: point source; psneb: point source in nebulosity; neb: nebulous;

HII: IRAC colors reminiscent of HII regions; sat: saturated in IRAC band.

class II and class I objects as determined from their near- and mid-infrared colors. A few knots of emission have IRAC colors that are reminiscent of HII regions, though they have not been detected in the radio continuum.

\subsection{Individual sources}

The $12.5 \mu \mathrm{m}$ image is shown in Fig. 7 with the positions of the three UC HII regions detected in W75 N(B) 


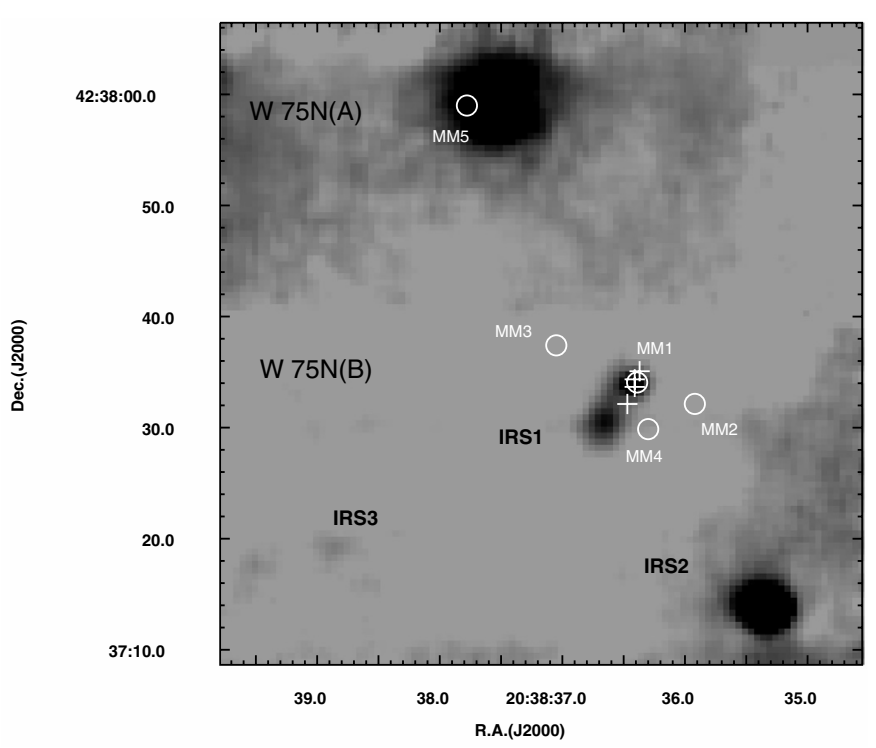

Fig. 7. Ground-based image of $\mathrm{W} 75 \mathrm{~N}$ at $12.5 \mu \mathrm{m}$ with the positions of the UC HII regions (plus), and the millimeter peaks (open circles) marked.

(plus symbols), the five millimeter cores (open circles) are indicated. The mid-infrared sources IRS 2, IRS 1 and IRS 3 show significant $2 \mu \mathrm{m}$ excesses. MM 1, detected only at 11.7 and $12.5 \mu \mathrm{m}$, is within the millimeter core, where some of the youngest objects of the cloud are located (Shepherd 2001). Our peak position of Table 1 identifies this source with the UC HII region VLA 3 (Torrelles et al. 1997). We did not detect this source at $18.7 \mu \mathrm{m}$ probably because of the lower sensitivity at this wavelength. Finally, the other millimeter cores (MM 2, MM 3 and MM 4) reported in the region were not detected in the mid-infrared, either by IRAC or in our ground-based survey (cf. Figs. 5 and 7), indicating that these objects are in the earliest stages of their life, still in the accretion phase. MIPS imaging of this region was severely saturated, preventing us from seeing any possible embedded IR core.

\subsection{Mid-infrared sources IRS 1, IRS 2 and IRS 3}

IRS 2 and IRS 3 were not resolved at any wavelength in the diffraction-limited mid-infrared images obtained from the ground with CID or from space with IRAC. In contrast, IRS 1 is resolved at all wavelengths, from 1.2 to $18.7 \mu \mathrm{m}$ on the 2MASS, IRAC and CID images, with measured sizes ( $F W H M$ in arcsec, $\mathrm{RA} \times \mathrm{Dec}): 5 \times 6$ in 1.2 and $1.6 \mu \mathrm{m}, 6 \times 9$ in 2.2 and $3.6 \mu \mathrm{m}, 7 \times 12$ in $4.5,5.8,8.0 \mu \mathrm{m}$, and $4 \times 5$ in the longer wavelengths. All these indicate the presence in IRS 1 of a symmetric dust envelope of size of around 0.05 to 0.1 parsec that scatters radiation from the central young stellar object. Probably, shocked gas emission contributes to the more extended distribution of IRS 1 seen in IRAC band $2(4.5 \mu \mathrm{m})$. The $2.12 \mu \mathrm{m} \mathrm{H}_{2}$ knot of emission reported by Davis et al. (1998) and Shepherd et al. (2003) 10" west of IRS 1 is not conspicuously bright in any of the IRAC bands (cf. Fig. 5) as it may be masked by diffuse nebulosity. The almost identical morphology seen at 2.2,3.6 and $4.5 \mu \mathrm{m}$ of the ring-like extended structure is

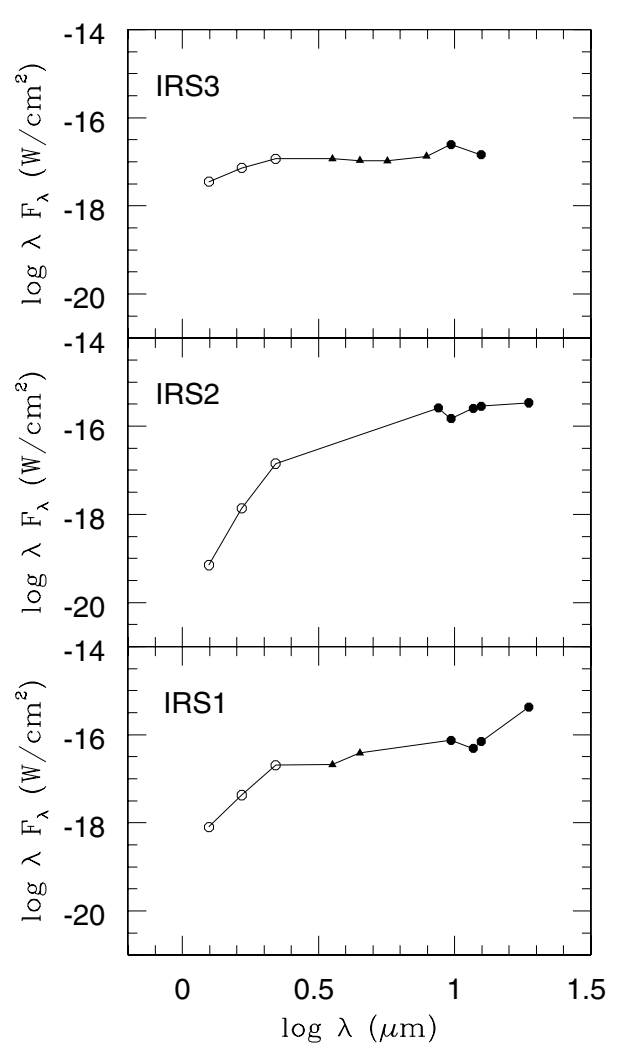

Fig. 8. Spectral energy distributions of IRS 1, IRS 2 and IRS 3. The near-infrared photometry (open circles) is from 2MASS. Triangles are IRAC fluxes and filled circles are from CID images.

Table 4. Infrared luminosities and spectra indices.

\begin{tabular}{lll}
\hline \hline Source & $\begin{array}{l}L_{\mathrm{IR}} \\
\left(L_{\odot}\right)\end{array}$ & $\alpha_{\mathrm{IR}}$ \\
\hline IRS 2 & 254 & 0.72 \\
IRS 1 & 540 & 1.72 \\
IRS 3 & 36 & 0.12 \\
\hline
\end{tabular}

seen within $20^{\prime \prime}$ SE of IRS 1, confirming the reflection nature of this radiation.

Combining the flux densities shown of Tables 1 and 2 with the 2MASS $J H K$ photometry, we obtained the spectral energy distribution (SED) of IRS 2, IRS 1 and IRS 3 illustrated in Fig. 8. IRS 3 shows a flat spectrum, while IRS 2 and IRS 1 have the silicate feature in absorption at $9.7 \mu \mathrm{m}$. IRS 2 shows a steeper spectrum than IRS1. We derived their infrared luminosities by integrating the spectra of Fig. 8 and the 2.2 and $12.5 \mu \mathrm{m}$ infrared spectral indices $\alpha_{\mathrm{IR}}=\mathrm{d} \log \lambda \mathrm{F}(\lambda) / \mathrm{d} \log \lambda$. These parameters are reported in Table 4.

\section{4. $M M 1$}

In W75 N(B) we have have detected for the first time a midinfrared source within the millimeter core MM 1, where some of the youngest sources of the cloud are located (Shepherd 2001). The source was unresolved by CID and IRAC at all wavelengths. We derived a set of physical parameters for this object by analyzing its SED, which is shown in Fig. 9; this 


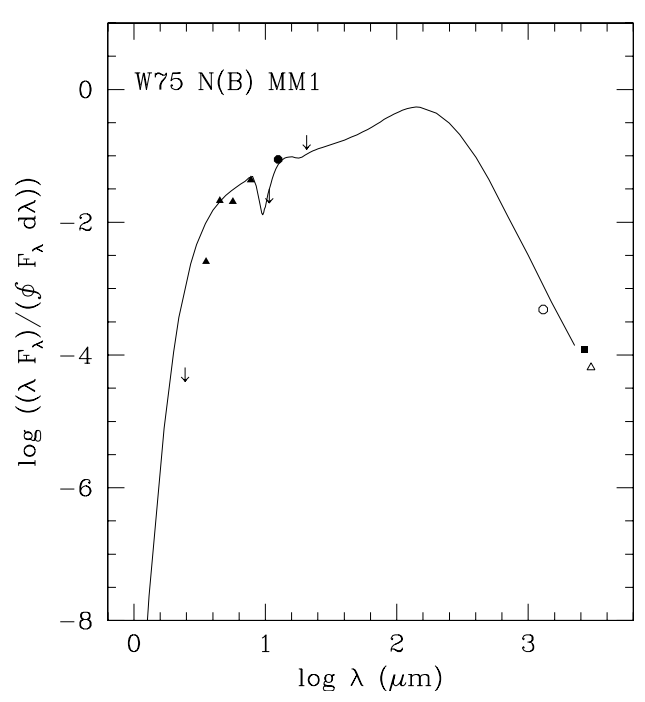

Fig. 9. Spectral energy distribution of the source at the position of MM 1. The upper limit at $2.2 \mu \mathrm{m}$ is from 2MASS. The solid line is the spherically symmetric dust model obtained from the DUSTY code with the parameters shown in Table 5 .

source is the most luminous and masssive star in the W75N(B) complex. The SED was compiled from the SPM mid-infrared flux densities of Table 1, the IRAC data of Table 2 and the 1.3, 2.7 and $3 \mathrm{~mm}$ flux densities reported by Shepherd (2001), Shepherd et al. (2003) and Watson et al. (2002). For the fit, we adopted a spherically symmetric dust envelope model (Ivezić \& Elitzur 1997) developed by the model code (Ivezić et al. 1999). We assumed standard optical properties for the dust grains (Draine \& Lee 1984), an early B-type central star of temperature $T_{\mathrm{c}}=15000 \mathrm{~K}$, a temperature of the inner radius $T_{\mathrm{i}}=1300 \mathrm{~K}$ close to the sublimation dust temperature and a dust density distribution of the type $n(r) \propto r^{-0.2}$ which provided the best fit. Table 5 reports the derived parameters, including the inner $\left(r_{\mathrm{i}}\right)$ and the outer radii $\left(r_{\mathrm{o}}\right)$ of the envelope and the dust temperature $\mathrm{T}_{d}$ at the outer radii. The solid line in Fig. 9 reproduces the fitted model. The derived luminosity of $\sim 750 L_{\odot}$ is consistent with the presence of B3-B5 ZAMS star deeply embedded in the MM 1 core.

Figure 5 shows (in green) the strong IRAC Band $2(4.5 \mu \mathrm{m})$ nebulous emission in the close vicinity of MM 1 . This is attributed to shocked lines (especially $\mathrm{H}_{2}$ ) that dominates this IRAC band. The complex image structure shows that that the shock, if produced by an outflow, is not produced by a particularly simple bipolar, collimated flow but rather by a more complex (perhaps even multiple) shock structure as also suggested by the distribution of blue shifted and red shifted $\mathrm{CO}$ emission in that region (Shepherd et al. 2003). Due to heavy extinction, the $2.12 \mu \mathrm{m} \mathrm{H}_{2}$ morphology (Shepherd et al. 2003) is not identical to that seen by IRAC. There is no other similar shock activity in the $\mathrm{W} 75 \mathrm{~N}$ region, a result that is consistent with the notion that the MM 1 protostar is considerably younger that the source in $\mathrm{W} 75 \mathrm{~N}(\mathrm{~A})$.
Table 5. Model parameters of MM 1 from the DUSTY code.

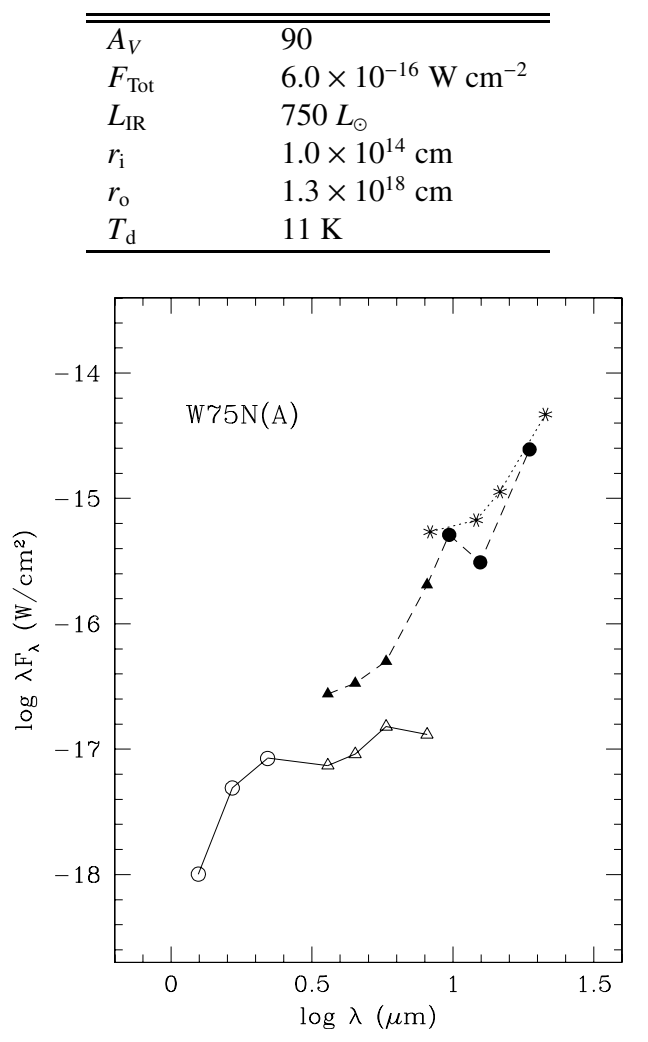

Fig. 10. Spectral energy distribution of W75 N(A). The near-infrared photometry is taken from 2MASS (open circles). Mid-infrared fluxes from the $M S X$ satellite are asterisks. Filled circles represent CID data and triangles, IRAC fluxes: open symbols refer to the central star and filled symbols to a large $\left(6^{\prime \prime}\right)$ aperture.

\section{5. $W 75 N(A)$}

The extended radio source $\mathrm{W} 75 \mathrm{~N}(\mathrm{~A})$ is associated with the millimeter source MM 5 (cf. Fig. 7). Its mid-infrared morphology, illustrated in Fig. 4 which presents the $18.7 \mu$ m emission contours overlaid on a RGB-coded image at 3.6, 5.8 and $8 \mu \mathrm{m}$, is very similar to that seen at $6 \mathrm{~cm}$ (cf. Fig. 6 of Shepherd et al. 2004). This clearly suggests a direct interaction between the ionized gas and the dust cloud.

The 1-20 $\mu \mathrm{m}$ spectral energy distribution of the diffuse HII region W75 N(A) is reported in Fig. 10. Our mid-infrared photometry obtained with an aperture of $6^{\prime \prime}$ is in agreement with the data taken from the MSX satellite. Integrating the SED, we obtained $L(1-20 \mu \mathrm{m})=1930 L_{\odot}$ or a total luminosity of around $4000 L_{\odot}$. This value is consistent with the presence of a B0.5 star, as also estimated by Shepherd et al. (2004) based on the required UV flux. The star seen at the center of the HII region at all infrared wavelengths has $J H K_{\mathrm{s}}$ colors that suggest that this early-B star is reddened by $A_{\mathrm{V}}=25$ with no significant $K$-band excess emission, though at $\lambda>4 \mu \mathrm{m}$, warm dust emission close to the star is evident. Nevertheless, the stellar absolute $K$ magnitude implied by the photometry is $M_{K}=-4.26$, at least one magnitude brighter than that expected for the ZAMS B0-B1 star that is needed for the HII region as observed in radio-continuum, and also by the total luminosity 
derived above. The reason for this discrepancy lies probably in uncertain $K$ photometry for this star in the 2MASS Point Source Catalog that gives a photometric quality flag "E" for this band, meaning a poor profile fit causing a low-quality measurement. One of the possibilities that might explain this is the presence of a cooler close companion that contributes significantly to the near-infrared flux but not to the UV flux. The extended emission, measured with a large aperture, at $\lambda>3 \mu \mathrm{m}$, however, is characteristic of dusty HII regions. Its overall characteristics presented here support previous claims that that this is the most highly evolved source of the complex, with an age between 1.2 and 5 million years (Shepherd et al. 2004).

\section{Conclusions}

We report ground-based mid-infrared images at 8,7, 9.7, 11.7, 12.5 and $18.7 \mu \mathrm{m}$ of the high-mass star forming region W75 N(B). Combining these observations with IRAC images at 3.6, 4.5, 5.8 and $8.0 \mu \mathrm{m}$ of the same region, the following conclusions we reached:

1) A young stellar cluster with at least 25 members in an area of $120^{\prime \prime} \times 120^{\prime \prime}$ around $\mathrm{W} 75 \mathrm{~N}(\mathrm{~B})$ has been found by analyzing the [3.6]-[4.5] versus [5.8]-[8.0] and the $H-K_{\mathrm{s}}$ versus $K_{\mathrm{s}}-$ [3.6] plots, obtained from 2MASS and IRAC photometry.

2) Four sources were detected at $12.5 \mu \mathrm{m}$. Three of these (see Fig. 1 and Table 1) were identified as the known young stellar objects IRS 2, IRS 1 and IRS 3 . IRS 2 is partially saturated in all the four IRAC bands and IRS 1 show a diffuse emission, partially saturated at its peak in two IRAC images. From their respective spectral energy distributions, we obtained the infrared luminosities and spectral indices of these sources (Table 4). IRS 1 and IRS 2 are probably early-B young stellar objects associated with dust envelopes, while IRS 3 , with a flattened spectral energy distribution, is a Class II intermediatemass young object. This is confirmed by the location of this source in the IRAC two-color diagram of Fig. 6.

3 ) In the youngest region, W75 N(B), which is also characterized by the presence of several ultracompact HII regions, continuum millimeter peaks and $\mathrm{H}_{2} \mathrm{O}$ masers (Shepherd et al. 2004), we have found a new mid-infrared source at the position of the millimeter core MM 1 and very close to the UC HII VLA 3. Mid-infrared images at higher spatial resolution are required to confirm this last identification. Physical parameters were derived for this source (Table 4) by fitting a spherically symmetric dust envelope to its SED. The derived bolometric luminosity of $\sim 750 L_{\odot}$ and the high value of $A_{\mathrm{V}}=90$ suggest that this is a young B1-B3 star deeply embedded and one of the youngest objects observed in W75 N(B).

4) The HII region $\mathrm{W} 75 \mathrm{~N}(\mathrm{~A})$ located in the northern part of the cloud shows a mid-infrared morphology very similar to that observed in the radio continuum. This indicates that dust and gas are well mixed within this spherically shaped HII region. The near-infrared colors of its central star and the computed infrared luminosity of the source confirm that the HII region is ionized by a single B0.5 star reddened by $A_{\mathrm{V}} \simeq 25$. With an estimated age of 2 to $5 \mathrm{Myr}$, this is the oldest object in the region.
5) The present observations support previous results that this region harbors young stellar objects with a wide age spread, from millimeter sources with no mid-infrared and radio counterparts to an evolved quasi-spherical HII region. Thus, the molecular cloud in $\mathrm{W} 75 \mathrm{~N}$ has been producing massive stars for the last several million years.

Acknowledgements. We thank M. Marengo for his valuable assistance in obtaining accurate IRAC fluxes for the partially saturated sources. We also thank Dr. Tom Megeath for suggestions that led to improvements of this paper. HAS acknowledges support from NASA Grant NAG5-10659. Part of this work was supported by a CNR(Italy)-Conacyt(Mexico) bilateral agreement. M.T. also acknowledges grants from Conacyt and DGAPA-UNAM (IN-102803). This paper is partially based on observations made with the Spitzer Space Telescope, which is operated by the Jet Propulsion Laboratory, California Institute of Technology (CIT) under National Aeronautics and Space Administration (NASA) contract 1407. The publication also makes use of data products from the Two Micron All Sky Survey, which is a joint project of the University of Massachusetts and the Infrared Processing and Analysis Center/CIT, funded by NASA and the National Science Foundation.

\section{References}

Allen, L. E., Calvet, N., D’Alessio, P., et al. 2004, ApJS, 154, 363

Baart, E. E., Cohen, R. J., Davies, R. D., et al. 1986, MNRAS, 219, 145

Davis, C. J., Moriarty-Schieven, G., Eislöffel, J., et al. 1998, AJ, 115, 118

Draine, B. T., \& Lee, H. M. 1984, ApJ, 284, 89

Fazio, G., Hora, J. L., Allen, L. E., et al. 2004, ApJS, 154, 10

Haschick, A. D., Reid, M. J., Burke, B. F., et al. 1981, ApJ, 244, 76

Hunter, T. R., Taylor, G. B., Felli, M., \& Tofani, G. 1994, A\&A, 284, 215

Indebetouw, R., Mathis, J. S., Babler, B. L., et al. 2005, ApJ, 619, 931

Ivezić, Z., \& Elitzur, M. 1997, MNRAS, 287, 799

Ivezić, Z., Nenkova, M., \& Elitzur, M. 1999, User Manual for Dusty (University of Kentucky), Internal Rep.

Jones, T. J., Woodward, M. L., Boyer, R. D., \& Polomsky, E. 2005, ApJ, 620, 731

Koornneef, J. 1983, A\&A, 128, 84

Marston, A. P., Reach, W. T., Noriega-Crespo, A., et al. 2004, ApJS, 154,333

Megeath, S. T., Allen, L. E., Guthermuth, R. A., et al. 2004, ApJS, 154,367

Moore, T. J. T., Mountain, C. M., \& Yamashita, T. 1991a, MNRAS, 248, 79

Moore, T. J. T., Mountain, C. M., Yamashita, T., \& Mc Lean, I. S. 1991b, MNRAS, 248, 377

Salas, L., Gutierrez, L., Tapia, M., et al. 2003, in Instrument Design and Performance for Optical/Infrared Ground-Based Telescopes, ed. I. Mesanori, \& A. F. M. Moorwood, SPIE Proc., 4841, 594

Shepherd, D. S. 2001, ApJ, 546, 345

Shepherd, D. S., Testi, L., \& Stark, D. P. 2003, ApJ, 584, 882

Shepherd, D. S., Kurtz, S. E., \& Testi, L. 2004, ApJ, 601, 952

Stetson, P. B. 1987, PASP, 99, 191

Tapia, M. 1981, MNRAS, 197, 949

Torrelles, J. M., Gómez, J. F., Rodriguez, L. F., et al. 1997, ApJ, 489, 744

Torrelles, J. M., Patel, N. A., Anglada, G., et al. 2003, ApJ, 598, L115

Watson, C., Churchwell, E., Pankonin, V., et al. 2002, ApJ, 577, 260

Werner, M. W., Roellig, T. L., Low, F. J., et al. 2004, ApJS, 154, 1 


\section{Online Material}


P. Persi et al.: Star formation in W75 N, Online Material p 2

\begin{tabular}{|c|c|c|c|c|c|c|c|c|c|}
\hline 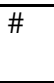 & $\begin{array}{l}\mathrm{RA}(2000) \\
\mathrm{h} \mathrm{m} \mathrm{s}\end{array}$ & $\begin{array}{l}\operatorname{Dec}(2000) \\
\circ, \prime \prime\end{array}$ & $\begin{array}{l}3.6 \\
\text { mag }\end{array}$ & $\begin{array}{l}4.5 \\
\mathrm{mag}\end{array}$ & $\begin{array}{l}5.8 \\
\mathrm{mag}\end{array}$ & $\begin{array}{l}8.0 \\
\mathrm{mag}\end{array}$ & $\begin{array}{l}J \\
\text { mag }\end{array}$ & $\begin{array}{l}H \\
\text { mag }\end{array}$ & $\begin{array}{l}K \mathrm{~s} \\
\mathrm{mag}\end{array}$ \\
\hline 1 & 203831.65 & 423833.5 & 12.18 & 11.46 & 10.68 & 9.46 & & & $15.14(.15)$ \\
\hline 2 & 203831.99 & 423740.1 & 12.17 & 11.67 & 11.12 & 10.44 & 15.51 & 13.84 & 13.00 \\
\hline 3 & 203832.36 & 423741.6 & 13.25 & 12.41 & 11.76 & 10.51 & & & \\
\hline 4 & 203833.04 & 423753.9 & 13.68 & 13.26 & 12.38 & & & & \\
\hline 5 & 203833.07 & 423716.0 & 13.13 & 12.48 & 12.07 & & & & \\
\hline 6 & 203833.15 & 42386.3 & 12.81 & 12.24 & 11.50 & 9.96 & & & $14.27(.11)$ \\
\hline 7 & 203833.39 & 423757.9 & 13.66 & 12.92 & 12.34 & & & 15.20 & $13.98(.09)$ \\
\hline 8 & 203833.43 & 423658.8 & 12.16 & 12.15 & 11.33 & & & & 13.31 \\
\hline 9 & 203833.49 & 42375.6 & 12.13 & 12.03 & 11.28 & & 15.72 & 13.96 & 13.12 \\
\hline 10 & 203833.55 & 423745.2 & 13.58 & 13.15 & & & & 15.37 & $14.52(.09)$ \\
\hline 11 & 203833.57 & 42383.1 & 12.10 & 11.85 & 11.21 & & 14.68 & 13.35 & 12.73 \\
\hline 12 & 203833.62 & 423726.5 & 11.66 & 10.77 & 9.97 & 9.04 & & 16.14 & $14.32(.07)$ \\
\hline 13 & 203833.64 & 423710.7 & 12.57 & 12.30 & & & & 15.77 & 14.58 \\
\hline 14 & 203833.68 & 423652.1 & 11.39 & 10.87 & 10.21 & 9.78 & & 14.36 & 12.97 \\
\hline 15 & 203833.70 & 423833.2 & 11.39 & 11.29 & 10.73 & & & 14.12 & 12.42 \\
\hline 16 & 203833.74 & 423752.8 & 13.43 & 12.69 & 12.05 & & & 15.56 & $14.53(.10)$ \\
\hline 17 & 203834.17 & 423756.9 & 11.45 & 10.94 & 10.27 & 10.01 & 16.79 & 14.15 & 12.89 \\
\hline 18 & 203834.23 & 423736.8 & 12.95 & 12.56 & & & & 15.10 & $14.33(.08)$ \\
\hline 19 & 203834.24 & 42372.4 & 12.41 & 11.58 & & & & & $15.00(.23)$ \\
\hline 20 & 203834.33 & 423717.8 & 12.56 & 12.00 & & 9.03 & 15.99 & 14.08 & 13.15 \\
\hline 21 & 203834.34 & 423724.0 & 13.80 & 12.14 & & & & & \\
\hline 22 & 203834.44 & 423818.2 & 13.51 & 13.70 & & & 14.67 & 14.05 & 13.84 \\
\hline 23 & 203834.54 & 42386.7 & 12.47 & 11.71 & 10.92 & 9.29 & & & $14.94(.12)$ \\
\hline 24 & 203834.64 & 42377.6 & 10.01 & 9.55 & 7.78 & 6.14 & & 14.55 & $12.58(.09)$ \\
\hline 25 & 203834.67 & 423751.0 & 14.24 & 13.61 & 10.70 & & & & \\
\hline 26 & 203834.70 & 423741.2 & 13.59 & & & & & & \\
\hline 28 & 203834.92 & 423828.7 & 13.19 & 12.49 & 11.85 & 9.64 & & 16.00 & $14.77(.10)$ \\
\hline 29 & 203834.93 & 423645.1 & 13.37 & 12.52 & 11.54 & & & & \\
\hline 30 & 203834.98 & 423826.7 & 13.31 & 12.47 & & & & & \\
\hline 31 & 203835.10 & 423812.3 & 14.17 & 13.35 & 11.69 & & & & \\
\hline 32 & 203835.29 & 423649.1 & 11.57 & 10.88 & 10.11 & 10.03 & & & 13.60 \\
\hline 33 & 203835.30 & 423739.0 & 14.16 & 13.15 & & & & & \\
\hline 34 & 203835.31 & 423833.7 & 12.72 & 12.18 & 11.88 & 9.92 & & 15.43 & $14.14(.08)$ \\
\hline 35 & 203835.43 & 42387.1 & 13.02 & 12.45 & 10.33 & & & & \\
\hline 36 & 203835.55 & 42381.3 & 12.34 & 12.18 & 11.81 & & & & \\
\hline 37 & 203835.81 & 423749.5 & 10.84 & 9.76 & 8.11 & 6.82 & & & \\
\hline 38 & 203835.94 & 423829.9 & 13.78 & 12.91 & 11.07 & & & & \\
\hline 39 & 203836.22 & 423650.4 & 13.75 & 12.76 & & & & & \\
\hline 40 & 203836.27 & 423824.9 & 11.74 & 11.23 & 10.37 & & & 14.97 & 13.15 \\
\hline 41 & 203836.34 & 423811.9 & 11.33 & 10.76 & 10.25 & 10.25 & & & \\
\hline 42 & 203836.42 & 423726.1 & 8.18 & 6.80 & 5.87 & 3.52 & & 13.53 & $10.69(.11)$ \\
\hline 43 & 203836.47 & 423646.1 & 13.29 & 12.72 & & & & & \\
\hline 44 & 203836.49 & 423831.3 & 12.94 & 12.27 & 11.10 & 10.09 & & & $14.63(.09)$ \\
\hline 45 & 203836.54 & 423758.4 & 10.25 & 9.79 & 7.09 & 5.17 & & & $14.37(.25)$ \\
\hline 46 & 203836.62 & 423815.5 & 11.69 & 11.38 & 11.12 & & & & 13.93 \\
\hline 47 & 203836.70 & 423728.8 & 7.60 & 6.22 & & & 14.11 & 11.84 & 9.78 \\
\hline 48 & 203836.96 & 423741.9 & 10.52 & 9.16 & & & & & $13.77(.18)$ \\
\hline 49 & 203837.02 & 423735.2 & 9.40 & 8.29 & 8.02 & & & & \\
\hline 50 & 203837.11 & 423718.8 & 8.26 & 7.67 & 7.49 & 7.26 & & & 13.38 \\
\hline 51 & 203837.56 & 423728.5 & 11.10 & 9.72 & & & & & $11.92(.19)$ \\
\hline 52 & 203837.58 & 423733.2 & 10.69 & 9.50 & & & & & $12.67(.19)$ \\
\hline 53 & 203837.76 & 423758.1 & 8.73 & 7.79 & 6.50 & 5.63 & 13.95 & 11.46 & 10.07 \\
\hline 54 & 203837.83 & 423718.3 & 9.41 & 8.73 & & & & & $11.24(.11)$ \\
\hline 55 & 203837.82 & 423828.8 & 12.63 & 12.08 & 11.14 & 10.73 & & 15.90 & $14.68(.10)$ \\
\hline 56 & 203838.19 & 423733.3 & 10.82 & 9.78 & 8.73 & & & & $13.97(.17)$ \\
\hline 57 & 203838.40 & 42371.1 & 13.17 & 13.06 & & & 16.70 & 15.06 & $14.30(.16)$ \\
\hline 58 & 203838.43 & 423716.0 & 10.40 & 10.12 & & & & & \\
\hline 59 & 203838.74 & 423836.2 & 13.26 & 13.54 & & & & 15.96 & $15.06(.14)$ \\
\hline 60 & 203838.75 & 423812.5 & 11.21 & 10.44 & 10.68 & & & 14.71 & $12.67(.08)$ \\
\hline 61 & 203838.88 & 423716.9 & 8.23 & 7.66 & 6.90 & 5.65 & 12.61 & 10.98 & 9.72 \\
\hline
\end{tabular}


P. Persi et al.: Star formation in W75 N, Online Material $p 3$

\begin{tabular}{llllllllll}
\hline \hline$\#$ & $\begin{array}{l}\text { RA(2000) } \\
\text { h m s }\end{array}$ & $\begin{array}{l}\text { Dec(2000) } \\
{ }^{\prime},{ }^{\prime}\end{array}$ & $\begin{array}{l}3.6 \\
\text { mag }\end{array}$ & $\begin{array}{l}4.5 \\
\text { mag }\end{array}$ & $\begin{array}{l}5.8 \\
\text { mag }\end{array}$ & $\begin{array}{l}8.0 \\
\text { mag }\end{array}$ & $\begin{array}{l}J \\
\text { mag }\end{array}$ & $\begin{array}{l}H \\
\text { mag }\end{array}$ & $\begin{array}{l}K \mathrm{~s} \\
\text { mag }\end{array}$ \\
\hline 62 & 203838.91 & 423742.6 & 10.27 & 9.53 & 8.93 & 7.92 & 17.08 & 13.67 & 12.13 \\
63 & 203839.20 & 423839.8 & 12.43 & 12.28 & & & & & 13.50 \\
64 & 203839.25 & 42386.3 & 13.23 & 12.28 & & & & 15.37 & $13.70(.12)$ \\
65 & 203839.43 & 423710.4 & 10.94 & 10.30 & 9.23 & 8.36 & 16.29 & 13.66 & $12.26(.08)$ \\
66 & 203839.50 & 423757.6 & 10.96 & 10.86 & & & & & \\
67 & 203840.09 & 42388.0 & 11.36 & 11.14 & 10.51 & & 16.13 & 14.23 & 13.22 \\
68 & 203840.25 & 423742.8 & 13.12 & 12.17 & & & & & \\
69 & 203840.35 & 423650.9 & 13.29 & 13.19 & 12.49 & & 16.14 & 14.79 & $14.00(.11)$ \\
70 & 203840.60 & 42385.5 & 12.27 & 11.87 & & & 15.95 & 13.75 & 12.91 \\
71 & 203840.71 & 423835.3 & 12.48 & 12.09 & 10.72 & & & & $15.53(.13)$ \\
72 & 203840.81 & 423757.8 & 12.00 & 12.28 & & & & & \\
73 & 203840.90 & 423838.9 & 12.66 & 12.33 & 10.27 & 8.49 & & & $14.60(.11)$ \\
74 & 203841.25 & 423738.9 & 12.39 & 11.33 & 9.23 & 7.99 & & & \\
75 & 203841.62 & 423727.0 & 13.23 & 12.59 & & & & & \\
\hline
\end{tabular}

